\title{
The complete far infrared spectroscopic survey of Herbig AeBe stars obtained by ISO-LWS
}

\author{
D. Lorenzetti ${ }^{1}$, T. Giannini ${ }^{1}$, B. Nisini ${ }^{1}$, M. Benedettini ${ }^{2}$, D. Elia ${ }^{3}$, L. Campeggio ${ }^{3}$, and F. Strafella ${ }^{3}$ \\ 1 INAF - Osservatorio Astronomico di Roma, via Frascati 33, 00040 Monte Porzio, Italy \\ e-mail: teresa, bruni@coma.mporzio.astro.it \\ 2 Istituto di Fisica Spazio Interplanetario - CNR Area Ricerca Tor Vergata, via Fosso del Cavaliere, 00133 Roma, Italy \\ e-mail: milena@ifsi.rm.cnr.it \\ ${ }^{3}$ Università degli Studi di Lecce - Dipartimento di Fisica, via Arnesano, 73100 Lecce, Italy \\ e-mail: eliad, campeggio, strafella@le.infn.it
}

Received 17 May 2002 / Accepted 28 June 2002

\begin{abstract}
The ISO-LWS archive has been systematically searched in order to obtain a complete far IR spectrophotometric survey of Herbig AeBe (HAEBE) stars. The investigated sample is constituted by 15 objects which, together with the 11 HAEBE we have published in two previous papers, represents about $25 \%$ of all the known HAEBE. This catalogue constitues an essential data-base in view of far IR forthcoming space missions (e.g. Herschel Space Observatory), whose scientific programs are now in the planning phase. The new sources are analysed following the same approach as in our previous papers and both differences and similarities are discussed in a coherent framework. The [OI] $63 \mu \mathrm{m}$ and the [CII] $158 \mu \mathrm{m}$ lines are observed in many of the investigated sources, while the [OI] $145 \mu \mathrm{m}$ remains often undetected, due to its relative faintness. Molecular lines, in form of CO high- $J$ rotational transitions are detected in only three cases and appear associated to local density peaks. A new class of ISO-LWS spectra of HAEBE emerges, constituted by objects without any detected gas feature either in emission or in absorption. Not unexpectedly, these HAEBE are isolated from molecular clouds and, as such, lack of the cold circumstellar material probed by far IR ionic and molecular transitions. By comparing line intensity ratios with model predictions we find that photodissociation caused by the stellar photons and active in a clumpy medium is likely the prevalent excitation mechanism for the far IR lines. Finally, an evolutionary trend is found according to which the contribution of the far IR line emission to the total emitted energy is less and less important with time.
\end{abstract}

Key words. stars: circumstellar matter - stars: pre-main sequence - infrared: ISM - ISM: lines and bands - infrared: stars

\section{Introduction}

Most of the recent studies on HAEBE stars deal with their circumstellar environments, where large scale phenomena (e.g. outflows, jets, heating and cooling of the circumstellar material) take place and IR excess emission, above that of the photosphere, originates. A significant fraction of the HAEBE's emitted power is released in the far IR range that is inaccessible from the ground (i.e. $45-200 \mu \mathrm{m}$ ) and that contains many emission lines which are unique tracers of the circumstellar gas physical conditions. The first spectrophotometric survey of HAEBE stars in this range has been obtained thanks to the ISO Long Wavelength Spectrometer (LWS) observations (Lorenzetti et al. 1999; Giannini et al. 1999; Papers I and II, respectively). These spectra show that [OI] $63 \mu \mathrm{m}$, $145 \mu \mathrm{m}$ and [CII] $158 \mu \mathrm{m}$ are by far the strongest emission lines so that they have been used to diagnose, as dominant excitation mechanism, the photodissociation supported by

Send offprint requests to: D. Lorenzetti, e-mail: dloren@coma.mporzio.astro.it stellar more than interstellar photons. Molecular emission, in form of $\mathrm{OH}$ and $\mathrm{CO}$ transitions, with no evidence of water vapour emission, has been detected only toward those sources with high density $\left(n \gtrsim 10^{6} \mathrm{~cm}^{-3}\right)$ circumstellar material. Again, Photon Dissociated Regions (PDRs) models, allowing for some clumpiness (Burton et al. 1990), offer a satisfactory explanation for the molecular data, providing values for density $(n)$ and radiation field $\left(G_{0}\right)$ comparable to those derived from far IR ionic transitions.

Few other regions around HAEBE have been studied by means of ISO-LWS: the Cha II dark cloud (Nisini et al. 1996); the $\mathrm{BD}+40^{\circ} 4124$ group (van den Ancker et al. 2000); the NGC 7023 region (Fuente et al. 2000); and He 3-672 (Malfait et al. 1998). All the results from these papers, dedicated to discuss specific properties of the investigated regions, are not in contrast with the above findings.

More recently the LWS spectra of six additional HAEBE stars (nearly all with A spectral types) have been investigated by Creech-Eakman et al. (2002). These authors provide an interpretation of the far IR continua in terms of passively heated, 
flared, circumstellar disks. Their line analysis confirms our prior conclusions about the prevailing excitation mechanisms, but in almost all cases ( 5 out of 6 ) they report the unprecedented detection of $\mathrm{HI}$ and $\mathrm{HeII}$ recombination lines.

A large number of HAEBE stars has been also investigated with the ISO Short Wavelength Spectrometer (SWS) covering the 3-45 $\mu \mathrm{m}$ range (see: Waelkens et al. 1996; Malfait et al. 1998; Wesselius et al. 1996; van den Ancker et al. 2000; Thi et al. 2001; Benedettini et al. 1998, 2001; Meeus et al. 2001). SWS samples much more compact scales (from 10 to $20^{\prime \prime}$ ) compared to the largest LWS field of view $\left(\sim 80^{\prime \prime}\right)$, therefore the two instruments investigate the circumstellar gas in substantially different conditions.

This paper aims to complete and extend our previous analysis to all the far IR spectra of HAEBE stars through an unbiased search from the ISO archive, in order to derive the far IR behaviour of HAEBE as a class. It is worthwhile noting that the same spectral range will be investigated at a much higher spec$\operatorname{tral}\left(\mathcal{R} \simeq 10^{7}\right)$ and spatial $\left(\sim 9^{\prime \prime}\right)$ resolution with the instruments on board Herschel (Pilbratt 2001), hence the results of this spectroscopic survey can be considered as a useful database for planning future far IR observations.

\section{Selected sample}

We have searched the ISO archive (http://www. iso.vilspa.esa.es/ida/index_us.html) for LWS grating spectra of the objects listed in the most complete HAEBE stars compilation available to date (Thé et al. 1994, their Table 1). The archive search was simply made by centering a circular box on the HAEBE coordinates, whose size is the same of the LWS field of view $\left(80^{\prime \prime}\right)$. We have found LWS spectra of 26 HAEBE out of the 108 catalogued objects. In Papers I and II we have presented the LWS data of 11 HAEBE along with two spectroscopic far IR maps (NGC 7129 and MWC 1080): these observations constituted part of the ISO guaranteed time program and, as such, they were obtained adopting a coherent strategy (e.g. selection criteria for sources; exposure times; ON and OFF source measurements for background evaluation). In the following we present LWS observations of the remaining 15 objects which belonged to different proposals which had different aims. Including these objects allows to more than double the so far available sample, thus achieving a significant coverage $(\sim 25 \%)$ of all the known HAEBE stars. It is remarkable that, despite the random selection criteria, the final sample spans a wide range of parameters such as the spectral type (from A8 to O7), the bolometric luminosity (from 5 to $1.3 \times 10^{5} L_{\odot}$ ), the circumstellar extinction (from 0.2 to $11 \mathrm{mag}$ of visual extinction) and the associated outflow activity. The 15 new objects are listed in Table 1: the astrophysical parameters (distance, luminosity, $A_{V}$, etc.) have been taken by both Hipparcos (van den Ancker et al. 1998) and literature data; the parameter $r$ following the IRAS name indicates whether the optical source is within the IRAS ellipse (e) or, otherwise, it gives the angular distance (in arcsec) between the optical and the IRAS peak; a flag near the source name signals that some results from the LWS spectrum of that source have been already presented in the literature according to the reference indicated. For these flagged sources we will emphasize here new line detections (if any) and additional aspects not commented in the previous papers.

\section{Observations and data reduction}

The observations were carried out with the Long Wavelength Spectrometer (LWS: Clegg et al. 1996, Swinyard et al. 1996) on board the Infrared Space Observatory (ISO: Kessler et al. 1996) in full grating scan mode (LWS01 AOT). This configuration provides coverage of the 43-196.7 $\mu \mathrm{m}$ range at a resolution $\mathcal{R} \sim 200$, with an instrumental beam size of $\sim 80^{\prime \prime}$. The spectra were oversampled by a factor of 4 , were processed with the off-line pipeline (version 10) and reduced with the ISAP $^{1}$ (version 2.0). The flux calibration is based on observations of Uranus, resulting in an estimated accuracy of about $30 \%$ (Swinyard et al. 1996); the wavelength calibration accuracy is a small fraction $(\approx 25 \%)$ of the resolution element, i.e. $0.07 \mu \mathrm{m}$ in the range $43-90 \mu \mathrm{m}$ and $0.15 \mu \mathrm{m}$ in the range 90-196.7 $\mu \mathrm{m}$. The steps of the data reduction are: (i) averaging the different spectral scans after removing the glitches due to the impact of cosmic rays; (ii) correcting the low-frequency fringes which result from interference along the optical axis with off axis emission (Swinyard et al. 1996). In Table 2 the observation parameters are given: Cols. 2 to 7 provide the coordinates of the pointed position; the total integration time (Col. 8) is obtained by means of a number of subsequent scans (Col. 9); finally the date and the orbit numbers are given in Cols. 10 and 11 , respectively.

\section{Results}

The FIR spectra towards HAEBE stars consist of emission lines superimposed on a continuum. Discussion of both the continuum shape and a quantitative comparison with IRAS-PSC data, is reported in Elia et al. (2002). Figures 1-4 show the portions of the continuum subtracted spectra where lines with $S / N$ ratio $\geq 3$ have been detected; lines already presented in the previous literature are not replotted here.

The line analysis was performed on the defringed single detector spectra after subtracting a polynomial function which fitted the continuum and then using a single Gaussian function to fit the line profile. The errors on the line intensities correspond to a $1 \sigma$ statistical uncertainties derived from the rms fluctuations adjacent to the line. The criteria adopted for line detection are the following: $i)$ signal to noise ratio $S / N \geq 3$; ii) distance between observed and rest wavelength comparable to the wavelength calibration accuracy; iii) line width compatible with the nominal value of the relative detector $(\approx 0.30$ and $0.60 \mu \mathrm{m})$. The fluxes of the detected lines are given in Table 3 .

By inspecting Table 3 we realize that, as expected, the spectra are dominated by the presence of fine structure lines of both [OI] and [CII] with additional ionic contributions

1 The ISO Spectral Analysis Package is a joint development by the LWS and SWS Instrument teams and Data Center. Contributing Institutes are CESR, IAS, IPAC, MPE, RAL and SRON. 
Table 1. Parameters of the investigated HAEBE.

\begin{tabular}{|c|c|c|c|c|c|c|c|}
\hline Source & $\begin{array}{c}\text { Spectral } \\
\text { Type } \\
\end{array}$ & $\begin{array}{l}L_{\text {bol }} \\
\left(L_{\odot}\right) \\
\end{array}$ & $\begin{array}{c}A_{\mathrm{V}} \\
(\mathrm{mag})\end{array}$ & $\begin{array}{c}\text { Distance } \\
\text { (pc) }\end{array}$ & $\begin{array}{l}\text { IRAS } \\
\text { name }\end{array}$ & $\begin{array}{c}r \\
\left({ }^{\prime \prime}\right)\end{array}$ & Other identifications \\
\hline AB Aur ${ }^{a, b}$ & B9-A0 & 48 & 0.50 & 140 & $04525+3028$ & $\mathrm{e}$ & $\mathrm{BD}+30^{\circ} 741 / \mathrm{HD} 31293$ / MWC93 \\
\hline MWC $480^{a}$ & $\mathrm{~A} 2 / 3$ & 32 & 0.25 & 131 & $04555+2946$ & 26 & $\mathrm{BD}+29^{\circ} 774 / \mathrm{HD} 31648$ \\
\hline HD 34282 & $\mathrm{~A} 0 \mathrm{~V}$ & 4.8 & 0.59 & 160 & $05136-0951$ & $\mathrm{e}$ & $\mathrm{BD}-09^{\circ} 1110$ \\
\hline MWC $758^{a}$ & A3 & 22 & 0.22 & 200 & $05273+2517$ & $\mathrm{e}$ & $\mathrm{BD}+25^{\circ} 843 / \mathrm{HD} 36112$ \\
\hline CQ Tau ${ }^{a}$ & A8-F2 & 8 & 0.9 & 140 & $05328+2443$ & $\mathrm{e}$ & $\mathrm{BD}+24^{\circ} 873 / \mathrm{HD} 36910$ \\
\hline MWC 137 & B0 & $2.8 \times 10^{4}$ & 4.5 & 900 & $06158+1517$ & 13 & PN VV 42 / PK 195-00.1 \\
\hline He $3-672^{c}$ & B9V & 32 & 0.28 & 103 & $11312-6955$ & e & HD100546 / CPD-69²1557 \\
\hline He 3-741 & A4 & 35 & 0.31 & 116 & $11575-7754$ & $\mathrm{e}$ & HD104237 / CPD-770774 \\
\hline HD 141569 & $\mathrm{~A} 0 \mathrm{~V}$ & 32 & 0.47 & 99 & $15473-0346$ & $\mathrm{e}$ & $\mathrm{BD}-03^{\circ} 3833$ \\
\hline HD 142666 & $\mathrm{~A} 8 \mathrm{~V}$ & 10.7 & 0.71 & 116 & $15537-2153$ & $\mathrm{e}$ & BD $-21^{\circ} 4228 /$ CPD $-21^{\circ} 6063$ \\
\hline He 3-1141 & A7V-F0 & $>30$ & 0.56 & $>200$ & $16038-2735$ & $\mathrm{e}$ & HD144432 / CD-27º 10778 \\
\hline $\mathrm{TY}_{\mathrm{CrA}}^{d}$ & B9 & 98 & 1.5 & 140 & - & & $\mathrm{CPD}-37^{\circ} 8450 / \mathrm{CD}-37^{\circ} 13024$ \\
\hline $\mathrm{BD}+40^{\circ} 4124^{\dagger a, e}$ & $\mathrm{~B} 2 \mathrm{~V}$ & $3 \times 10^{3}$ & 3.0 & 1000 & - & & V1685 Cyg / He 3-1882 / MWC340 \\
\hline MWC $361^{f}$ & $\mathrm{~B} 2 / 3 \mathrm{~V}$ & $8 \times 10^{3}$ & 1.92 & 430 & - & & $\mathrm{HD} 200775 / \mathrm{BD}+67^{\circ} 1283$ \\
\hline $\mathrm{LkH} \alpha 233^{a}$ & A7 & $100-150$ & 2.6 & 800 & - & & V375 Lac / Mrk 914 \\
\hline
\end{tabular}

Notes to the Table:

${ }^{a}$ Creech-Eakman et al. (2002); ${ }^{b}$ Bouwman et al. (2000); ${ }^{c}$ Malfait et al. (1998); ${ }^{d}$ Corporon et al. (1999); ${ }^{e}$ van den Ancker et al. (2000); ${ }^{f}$ Fuente et al. (2000).

$\dagger$ the large LWS beam includes both $\mathrm{LkH} \alpha 224$ (an HAEBE star) and marginally LkH $\alpha 225$ (an active embedded YSO) whose contributions can be separated only on SWS observations (van den Ancker et al. 2000).

$r$ indicates the distance of the optical counterpart from the IRAS peak. Such distance is not given whenever the optical source lies within the IRAS uncertainty ellipse (e).

Table 2. Journal of observations.

\begin{tabular}{|c|c|c|c|c|c|c|c|c|c|c|}
\hline \multirow[t]{2}{*}{ Target } & \multicolumn{3}{|c|}{$\bar{\alpha} \alpha(2000.0)$} & \multicolumn{3}{|c|}{$\overline{\delta(2000.0)}$} & \multirow{2}{*}{$\begin{array}{l}t_{\text {int }} \\
\text { (s) }\end{array}$} & \multirow[t]{2}{*}{$\overline{n_{\text {scan }}}$} & \multirow[t]{2}{*}{ date } & \multirow[t]{2}{*}{ orbit } \\
\hline & h & $\mathrm{m}$ & $\mathrm{s}$ & 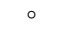 & & " & & & & \\
\hline AB Aur & 04 & 55 & 45.82 & +30 & 33 & 05.3 & 2530 & 15 & 27 Feb 98 & 835 \\
\hline MWC 480 & 04 & 58 & 46.07 & +29 & 50 & 37.3 & 2678 & 11 & $27 \mathrm{Feb} 98$ & 835 \\
\hline HD 34282 & 05 & 16 & 00.46 & -09 & 48 & 33.8 & 2680 & 16 & 27 Mar 98 & 863 \\
\hline MWC 758 & 05 & 30 & 27.35 & +25 & 19 & 58.0 & 2530 & 15 & 30 Mar 98 & 866 \\
\hline CQ Tau & 05 & 35 & 58.40 & +24 & 44 & 54.8 & 2080 & 12 & 15 Feb 98 & 823 \\
\hline MWC 137 & 06 & 18 & 44.83 & +15 & 16 & 43.6 & 1124 & 8 & 13 Mar 98 & 849 \\
\hline He 3-672 & 11 & 33 & 25.67 & -70 & 11 & 41.9 & 2228 & 4 & 29 Feb 96 & 104 \\
\hline He 3-741 & 12 & 00 & 05.07 & -78 & 11 & 33.7 & 3340 & 7 & 22 Jun 97 & 584 \\
\hline HD 141569 & 15 & 49 & 57.60 & -03 & 55 & 16.5 & 2228 & 13 & 04 Aug 97 & 627 \\
\hline HD 142666 & 15 & 56 & 40.08 & -22 & 01 & 40.9 & 2206 & 13 & 12 Feb 97 & 454 \\
\hline He 3-1141 & 16 & 06 & 58.02 & -27 & 43 & 10.0 & 2274 & 14 & 07 Sep 96 & 296 \\
\hline He $3-1141$ OFF & 16 & 06 & 55.93 & -27 & 48 & 31.5 & 2274 & 13 & 07 Sep 96 & 296 \\
\hline TY CrA & 19 & 01 & 40.68 & -36 & 52 & 32.6 & 2112 & 5 & 29 Oct 96 & 348 \\
\hline $\mathrm{BD}+40^{\circ} 4124$ & 20 & 20 & 28.30 & +41 & 21 & 51.5 & 2080 & 12 & 23 Dec 97 & 768 \\
\hline MWC 361 & 21 & 01 & 36.91 & +68 & 09 & 48.2 & 610 & 5 & 27 Oct 96 & 346 \\
\hline $\mathrm{LkH} \alpha 233$ & 22 & 34 & 40.91 & +40 & 40 & 04.6 & 2080 & 12 & 23 Dec 97 & 768 \\
\hline
\end{tabular}

(i.e. [OIII]) detected only toward the small $\mathrm{OB}$ association $\mathrm{BD}+40^{\circ} 4124$. Molecular emission, in form of high- $J$ CO rotational transitions, occurs in those cases where the column density $\left(A_{V}\right)$ is relatively large. Indeed the decreasing prevalence of molecular in favour of atomic line emission is a well recognized evolutionary trend, as already pointed out by Nisini et al. (2002), which is evident in going from the earliest phases of the protostellar evolution (Class 0 objects) toward Pre-Main Sequence stars (Class I/II objects). Molecular emission other than $\mathrm{CO}$ has been detected only in the spectrum associated to $\mathrm{BD}+40^{\circ} 4124$. It presents a feature around $179 \mu \mathrm{m}$ which can be attributed either to $\mathrm{H}_{2} \mathrm{O}(179.53 \mu \mathrm{m})$ or to $\mathrm{CH}^{+}(179.61 \mu \mathrm{m})$.
Such emission could result from the contamination due to the shocked gas in the outflow of LkH $\alpha 225$ (Palla et al. 1995).

By comparing our results with those already published by other authors we find the same values within the given 1 or $1.5 \sigma$ error. Minor exceptions are: (i) CQ Tau: we failed in detecting the [OI] $63 \mu \mathrm{m}$ line, found by Creech-Eakman et al. (2002) at a $S / N$ level of about 5; (ii) BD $+40^{\circ} 4124$ : conversely, here we report for the first time two more features at a significant level $(S / N \geq 5)$, namely [NII] $(122 \mu \mathrm{m})$ and $\mathrm{H}_{2} \mathrm{O}$ or $\mathrm{CH}^{+}(179 \mu \mathrm{m})$, not identified in the van den Ancker et al. (2000) spectrum. However, the relevant disagreement between our and previous results concerns the detection of HI (23-18), 
(18-16), (20-17), (22-16) and HeII (23-21), (25-23) and (25-24) lines found by Creech-Eakman et al. (2002) in five out of six investigated objects. Their important claim has motivated us to perform a careful analysis of those spectral segments, but we can neither confirm their detections nor give any clue for the presence, at those wavelengths, of spectral features which could be assigned to transitions other than HI and HeII. We have been able to recognize only one emission feature in CQ Tau at $60.41 \mu \mathrm{m}$ (with a $S / N \sim 2.5$ ), but we do not believe that helium recombination is plausible in view of the $10000 \mathrm{~K}$ effective temperature of an isolated and late A-type star such as CQ Tau. Searching the available spectral line catalogues, a plenty of transitions corresponding to that wavelength are found, but no clear identification can be however attributed to the observed feature.

In principle, an OFF source spectrum should be taken at a suitable distance from the target in order to derive line intensities as uncontaminated as possible by the parent cloud emission. An OFF spectrum is available only for He 3-1141 in the presented sample of sources. To find a reasonable method for evaluating possible OFF contaminations at different wavelengths the following has been considered. From the sample presented in Paper I we know that [OI] $63 \mu \mathrm{m}$ and [CII] $158 \mu \mathrm{m}$ are the lines commonly detected in both the ON and OFF spectra. Because of the higher excitation temperature, the [OI] line is definitively brigther $\mathrm{ON}$ source, while the [CII] line tends to have comparable intensity between $\mathrm{ON}$ and OFF pointings for the low luminosity sources, corresponding to low flux levels of $2-5 \times 10^{-20} \mathrm{~W} \mathrm{~cm}^{-2}$. This fact has been also confirmed by the OFF measurement of $\mathrm{He} 3-1141$, where the $[\mathrm{CII}]_{\mathrm{OFF}}$ flux is $4.1 \pm 0.5 \times 10^{-20} \mathrm{~W} \mathrm{~cm}^{-2}$, about the same value of the $\mathrm{ON}$ flux.

Creech-Eakman et al. (2002) performed an analysis of the background contamination around HAEBE stars using COBE, ISOPHOT and IRAS data reaching slightly different results. They found for all their stars (with the exception of $\left.\mathrm{BD}+40^{\circ} 4124\right)$ a contamination level in the $100<\lambda<240 \mu \mathrm{m}$ range less than $0.7 \times 10^{-20} \mathrm{~W} \mathrm{~cm}^{-2}$ in the LWS spectral resolution element (this value is about 13 times larger for $\left.\mathrm{BD}+40^{\circ} 4124\right)$. As a consequence, they did not apply any background correction to their data. Although neglecting or not the background contributions have minor implications on deriving the overall properties of the HAEBE stars, nevertheless we note how their estimate (when checked on the only available case) attributes to the $[\mathrm{CII}]$ diffuse emission a value more than a factor of five lower than the detected value.

Based on the above arguments: (i) a value of $2-5 \times$ $10^{-20} \mathrm{~W} \mathrm{~cm}^{-2}$ will be adopted in the following as the background contribution to the [CII] emission in regions around objects with A spectral type; (ii) a reduction by a factor of two will be applied to the high [CII] flux levels; (iii) no correction at all will be done to the [OI] emission. Only the case of $\mathrm{BD}+40^{\circ} 4124$ should be considered with some caveat.

The spectroscopic data given in Table 3 indicate that a subclass of HAEBE stars exists constituted by objects which show either no far IR emission line (MWC 480, HD 141569 and He 3-1141) or only small [CII] contributions marginally above the ubiquitous interstellar value (HD 34282, MWC 758, CQ Tau and HD 142666). The above mentioned HAEBE have an

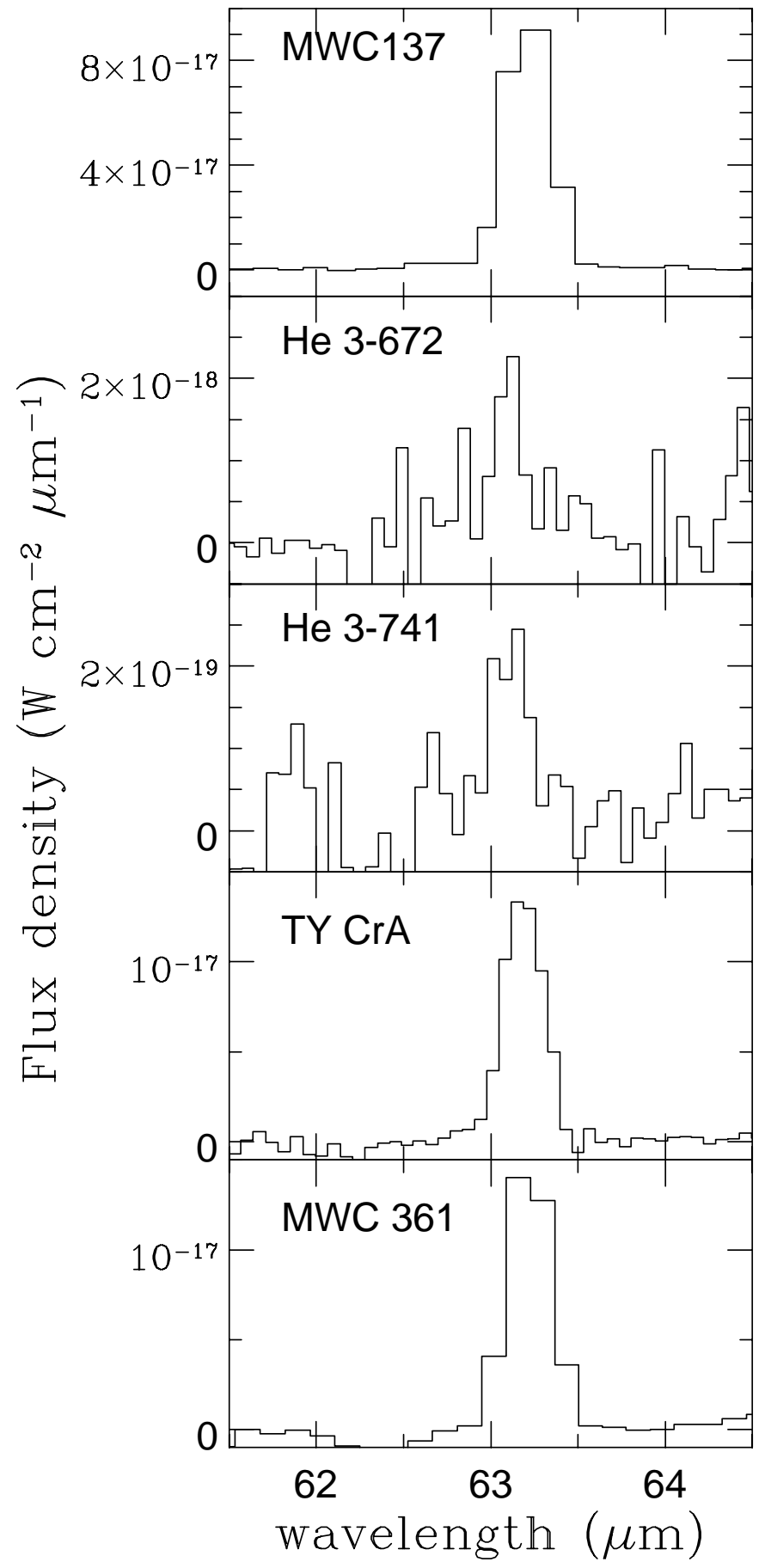

Fig. 1. Continuum subtracted LWS spectra of the investigated HAEBE stars: selected ranges containing the [OI] $63 \mu \mathrm{m}$ line. Lines already presented in the previous literature are not re-depicted here.

A-spectral type and an $A_{V}$ value less than unity, conditions which both inhibit the excitation of the cold circumstellar material by stellar photons. Basically these objects are the least luminous ones among our sample, having all $L_{\text {bol }} \lesssim 30 L_{\odot}$ (see Table 1). From an observational point of view this limit represents an important prescription for selecting targets of future space missions; the implications of this threshold will be discussed in the next section. 


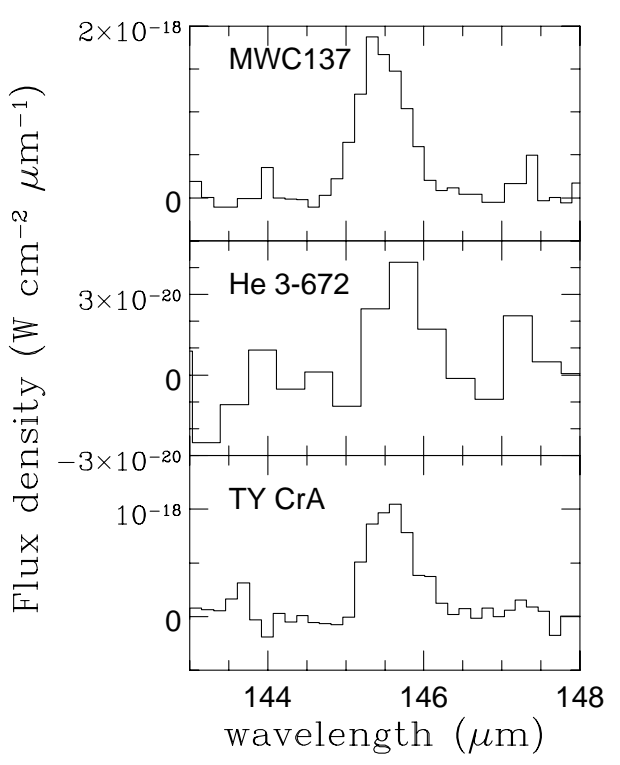

Fig. 2. As in Fig. 1 for the [OI] $145 \mu \mathrm{m}$ line. The spectrum of He 3672 has been re-binned at a lower resolution in order to increase the signal to noise ratio.
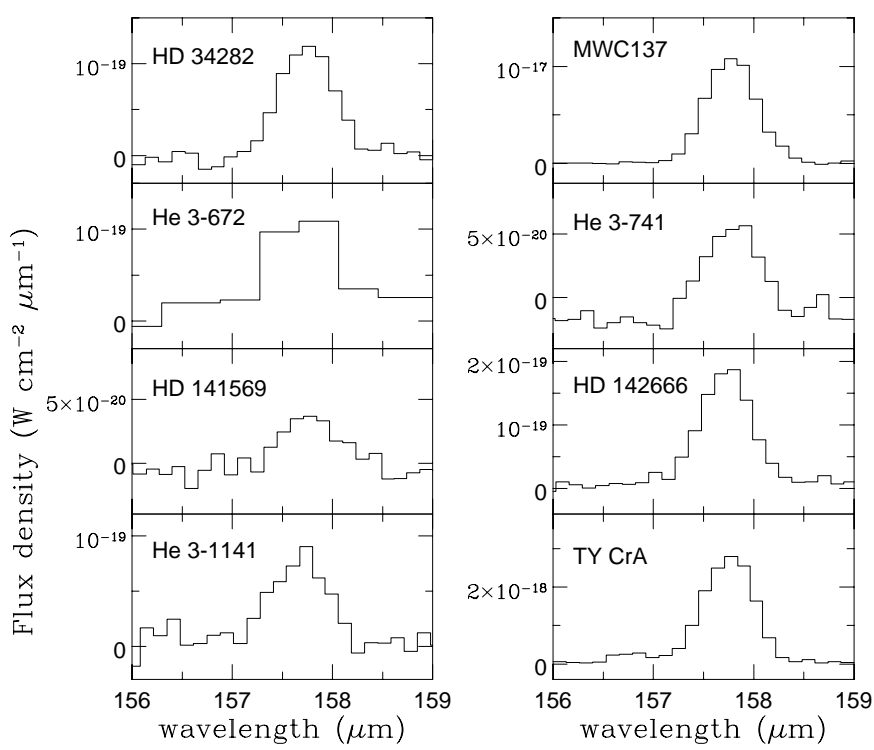

Fig. 3. As in Fig. 1 for the [CII] $158 \mu \mathrm{m}$ line. The spectrum of $\mathrm{He} 3-$ 672 has been re-binned at a lower resolution in order to increase the signal to noise ratio.

\section{Discussion}

\section{1. [OI] and [CII] lines}

The [OI] 63, $145 \mu \mathrm{m}$ and [CII] $158 \mu \mathrm{m}$ are the strongest features observed and have been used in Paper I as a diagnostic of the excitation mechanism and then of the physical conditions. Here we intend to follow the same method, namely to check whether or not the HAEBE of the present sample behave as the objects considered in Paper I. Line ratios and corresponding errors of the stars presented here are indicated in Fig. 5, where those already presented in Paper I are also reported for completeness but without any flag.

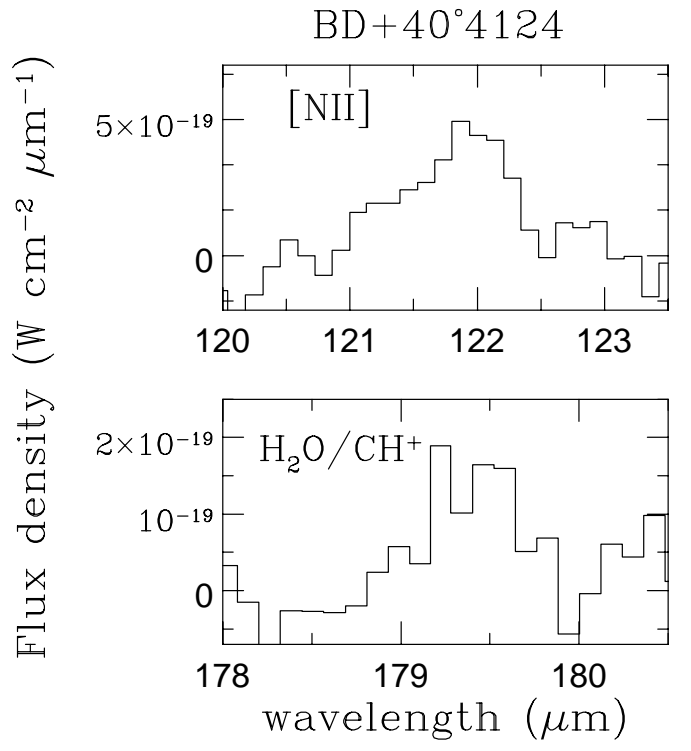

Fig. 4. As in Fig. 1 for the [NII] $122 \mu \mathrm{m}$ and $179 \mu \mathrm{m}$ emission feature (attributable to $\mathrm{H}_{2} \mathrm{O}$ or $\mathrm{CH}^{+}$). Both lines are detected for the first time in $\mathrm{BD}+40^{\circ} 4124$.

The observed line intensity ratios are superimposed in Fig. 5 on a grid of photodissociation models (Kaufman et al. 1999). The substantial difference between line intensity predictions of this model and those of other PDR models (e.g. Tielens \& Hollenbach 1985) is the inclusion of an additional heating: the ejection of photoelectrons from PAH and small grains. These latter are essential component of the HAEBE close environments, as demonstrated by many dedicated observations (see e.g. Meeus et al. (2001) and references therein). The extra heating source is essential because for a given value of density $(n)$ and radiation field $\left(G_{\mathrm{o}}\right)$, this model predicts increased OI and CII column densities, thus allowing to move at lower $n$ and $G_{\mathrm{o}}$ the transition point between CII dominated and OI dominated cooling. The location of the data points in Fig. 5, although in two cases (identified by the letters $c$ and e) not perfectly coincident with the model grid, gives further support to our previous interpretation in favour of phodissociation as dominating mechanism for far IR line excitation. Our hypothesis about the existence, around the HAEBE stars, of a PDR originated by stellar rather than interstellar FUV photons has received further support by the correlation between [CII] $158 \mu \mathrm{m}$ luminosity vs. the bolometric luminosity of the central source shown in Fig. 5 of Paper I. With reference to that plot, we note that the values $\left(L_{\mathrm{bol}}, L_{[\mathrm{CII}]}\right)$ of the new sources investigated here are perfectly aligned along the already presented relationship.

Alternative models do not seem so much promising: J-shock models (e.g. Hollenbach \& McKee 1989) can be ruled out, since they predict substantially different line ratios whose intersection with our PDR plane is indicated in Fig. 5 as the hatched area at the top right corner. Since a considerable fraction of the HAEBE is expected to drive molecular outflows, the role of non dissociative C-shocks (e.g. Draine et al. 1983) has been discussed in more detail in Paper I, where we conclude that possible contributions of C-shocks to the far IR emission 
Table 3. Line intensities of HAEBE stars.

\begin{tabular}{|c|c|c|c|}
\hline Source & $\begin{array}{c}\lambda_{\mathrm{obs}} \\
(\mu \mathrm{m})\end{array}$ & Identification & $F \pm \Delta F$ \\
\hline \multirow[t]{2}{*}{ AB Aur } & 63.18 & {$[\mathrm{OI}]{ }^{3} \mathrm{P}_{1}-{ }^{3} \mathrm{P}_{2}$} & $8 \pm 3^{a, b}$ \\
\hline & 157.77 & {$[\mathrm{CII}]{ }^{2} \mathrm{P}_{3 / 2}-{ }^{2} \mathrm{P}_{1 / 2}$} & $8.7 \pm 0.7$ \\
\hline MWC 480 & 157.77 & {$[\mathrm{CII}]{ }^{2} \mathrm{P}_{3 / 2}-{ }^{2} \mathrm{P}_{1 / 2}$} & $5.5 \pm 0.4$ \\
\hline HD 34282 & 157.74 & {$[\mathrm{CII}]{ }^{2} \mathrm{P}_{3 / 2}-{ }^{2} \mathrm{P}_{1 / 2}$} & $9.8 \pm 0.4$ \\
\hline MWC 758 & 157.79 & {$[\mathrm{CII}]{ }^{2} \mathrm{P}_{3 / 2}-{ }^{2} \mathrm{P}_{1 / 2}$} & $12.3 \pm 0.6$ \\
\hline CQ Tau & 157.75 & {$[\mathrm{CII}]{ }^{2} \mathrm{P}_{3 / 2}-{ }^{2} \mathrm{P}_{1 / 2}$} & $15.3 \pm 0.6$ \\
\hline \multirow[t]{3}{*}{ MWC 137} & 63.20 & {$[\mathrm{OI}]{ }^{3} \mathrm{P}_{1}-{ }^{3} \mathrm{P}_{2}$} & $3467 \pm 13$ \\
\hline & 145.46 & {$[\mathrm{OI}]{ }^{3} \mathrm{P}_{0}-{ }^{3} \mathrm{P}_{1}$} & $136 \pm 4$ \\
\hline & 157.77 & {$[\mathrm{CII}]{ }^{2} \mathrm{P}_{3 / 2}-{ }^{2} \mathrm{P}_{1 / 2}$} & $696 \pm 4$ \\
\hline \multirow[t]{3}{*}{ He 3-672 } & 63.13 & {$[\mathrm{OI}]{ }^{3} \mathrm{P}_{1}-{ }^{3} \mathrm{P}_{0}$} & $66 \pm 13$ \\
\hline & $145.70^{c}$ & {$[\mathrm{OI}]{ }^{3} \mathrm{P}_{1}-{ }^{3} \mathrm{P}_{0}$} & $3.2 \pm 0.8$ \\
\hline & 157.72 & {$[\mathrm{CII}]{ }^{2} \mathrm{P}_{3 / 2}-{ }^{2} \mathrm{P}_{1 / 2}$} & $11 \pm 1$ \\
\hline \multirow[t]{2}{*}{ He 3-741 } & $63.10^{c}$ & {$[\mathrm{OI}]{ }^{3} \mathrm{P}_{1}-{ }^{3} \mathrm{P}_{2}$} & $6 \pm 1$ \\
\hline & 157.79 & {$[\mathrm{CII}]{ }^{2} \mathrm{P}_{3 / 2}-{ }^{2} \mathrm{P}_{1 / 2}$} & $4.4 \pm 0.4$ \\
\hline HD 141569 & 157.75 & {$[\mathrm{CIII}]{ }^{2} \mathrm{P}_{3 / 2}-{ }^{2} \mathrm{P}_{1 / 2}$} & $3.8 \pm 0.5$ \\
\hline HD 142666 & 157.72 & {$[\mathrm{CII}]{ }^{2} \mathrm{P}_{3 / 2}-{ }^{2} \mathrm{P}_{1 / 2}$} & $10.7 \pm 0.5$ \\
\hline He 3-1141 & 157.72 & {$[\mathrm{CII}]{ }^{2} \mathrm{P}_{3 / 2}-{ }^{2} \mathrm{P}_{1 / 2}$} & $4.4 \pm 0.3$ \\
\hline \multirow[t]{5}{*}{ TY CrA } & 63.18 & {$[\mathrm{OI}]{ }^{3} \mathrm{P}_{1}-{ }^{3} \mathrm{P}_{2}$} & $405 \pm 14$ \\
\hline & 145.57 & {$[\mathrm{OI}]{ }^{3} \mathrm{P}_{0}-{ }^{3} \mathrm{P}_{1}$} & $79.0 \pm 0.6$ \\
\hline & $153.11^{c}$ & CO 17-16 & $7 \pm 3^{a}$ \\
\hline & 157.75 & {$[\mathrm{CII}]{ }^{2} \mathrm{P}_{3 / 2}-{ }^{2} \mathrm{P}_{1 / 2}$} & $173 \pm 3$ \\
\hline & $163.00^{c}$ & CO 16-15 & $6 \pm 3^{a}$ \\
\hline \multirow[t]{12}{*}{$\mathrm{BD}+40^{\circ} 4124$} & 51.84 & {$[\mathrm{OIII}]{ }^{3} \mathrm{P}_{2}-{ }^{3} \mathrm{P}_{1}$} & $53 \pm 8$ \\
\hline & 63.17 & {$[\mathrm{OI}]{ }^{3} \mathrm{P}_{1}-{ }^{3} \mathrm{P}_{2}$} & $736 \pm 7$ \\
\hline & 88.39 & {$[\mathrm{OIII}]{ }^{3} \mathrm{P}_{1}-{ }^{3} \mathrm{P}_{0}$} & $79 \pm 7$ \\
\hline & 121.91 & {$[\mathrm{NII}]{ }^{3} \mathrm{P}_{2}-{ }^{3} \mathrm{P}_{1}$} & $35 \pm 5^{b}$ \\
\hline & 145.50 & {$[\mathrm{OI}]{ }^{3} \mathrm{P}_{0}-{ }^{3} \mathrm{P}_{1}$} & $51 \pm 4$ \\
\hline & 153.36 & CO 17-16 & $13 \pm 4$ \\
\hline & 157.73 & {$[\mathrm{CII}]{ }^{2} \mathrm{P}_{3 / 2}-{ }^{2} \mathrm{P}_{1 / 2}$} & $560 \pm 4$ \\
\hline & 162.83 & CO 16-15 & $21 \pm 3$ \\
\hline & 173.52 & CO 15-14 & $27 \pm 4$ \\
\hline & 179.44 & $\mathrm{H}_{2} \mathrm{O} 2_{12}-1_{01}$ & $16 \pm 4$ \\
\hline & & $\mathrm{CH}^{+} 2-1$ & \\
\hline & 185.87 & CO 14-13 & $13 \pm 3$ \\
\hline \multirow[t]{4}{*}{ MWC361 } & 63.21 & {$[\mathrm{OI}]{ }^{3} \mathrm{P}_{1}-{ }^{3} \mathrm{P}_{2}$} & $504 \pm 15$ \\
\hline & 145.63 & {$[\mathrm{OI}]{ }^{3} \mathrm{P}_{0}-{ }^{3} \mathrm{P}_{1}$} & $65 \pm 1$ \\
\hline & 157.76 & {$[\mathrm{CII}]{ }^{2} \mathrm{P}_{3 / 2}-{ }^{2} \mathrm{P}_{1 / 2}$} & $285 \pm 3$ \\
\hline & $162.65^{c}$ & CO 16-15 & $7 \pm 3^{a}$ \\
\hline \multirow[t]{3}{*}{ LkH $\alpha 233$} & 63.20 & {$[\mathrm{OI}]{ }^{3} \mathrm{P}_{1}-{ }^{3} \mathrm{P}_{2}$} & $19 \pm 3$ \\
\hline & 145.60 & {$[\mathrm{OI}]{ }^{3} \mathrm{P}_{0}-{ }^{3} \mathrm{P}_{1}$} & $2.9 \pm 0.9$ \\
\hline & 157.74 & {$[\mathrm{CII}]{ }^{2} \mathrm{P}_{3 / 2}-{ }^{2} \mathrm{P}_{1 / 2}$} & $45.4 \pm 0.9$ \\
\hline
\end{tabular}

Notes: ${ }^{a} S / N$ between 2 and $3 ;{ }^{b} F W H M$ smaller or larger than instrumental; ${ }^{c}$ distance between observed and rest wavelength marginally exceeding the resolution element. Fluxes are expressed in $10^{-20} \mathrm{~W} \mathrm{~cm}^{-2}$.

lines cannot be ruled out, but such mechanism can be considered as the main responsible for the gas excitation just in a narrow region of the parameter space (shock speed, magnetic field, pre-shock density).

The plot depicted in Fig. 5 has to be regarded as a useful tool to assess the prevailing excitation mechanism for the HAEBE class of objects, but cannot be used for finely deriving the physical parameters of the associated PDRs. Even in those cases where the small errors would formally allow such a derivation, the values are to be considered with some caution. Possible self-absorption of [OI] $63 \mu \mathrm{m}$ or thickness effects on both the [OI] lines discussed below (Sect. 5.4), tend to make uncertain the definition of the PDR parameters. In the next section a more reliable diagnostic, based also on molecular line emission, will be discussed.

\subsection{Molecular emission}

Out of the 15 considered HAEBE, we found molecular emission in form of $\mathrm{CO}$ rotational transitions in three objects: TY CrA, BD $+40^{\circ} 4124$ and MWC 361; hence the detection rate of molecular emission estimated on the overall sample is about $25 \%$ (6 out 26 objects). Although molecular lines are usually weak (see Table 3 and Paper II), we do not believe the lack of detection in other sources is due only to an instrumental sensitivity limit, but it is also related to an intrinsic property of the circumstellar environment, namely the existence or not of some compact density peaks near the source where the column density is expected to substantially increase. This occurrence has been already discussed in Paper II and our aim is to check this point on the three objects considered here.

To do that we have calculated the CO luminosity starting from the detected rotational lines, using our Large Velocity Gradient (LVG) code (see Paper II for details) to solve the equations of the statistical equilibrium for the level population. The total CO luminosity $\left(L_{\mathrm{CO}}\right)$ is derived by summing up all the intensities predicted by the model, while the associated uncertainty of about $30 \%$ stems from the spread among the best 30 models. Once $L_{\mathrm{CO}}$ is obtained for each source, we have plotted $L_{\mathrm{CO}} / L_{[\mathrm{O}][145}$ vs. $L_{\mathrm{CO}} / L_{[\mathrm{CII}]}$ in Fig. 6 along with the clumpy PDR model predictions by Burton et al. (1990). This plot offers the advantage of providing a diagnostic of the physical conditions by means of both atomic and molecular line emission; moreover it uses transitions not affected (as the [OI] $63 \mu \mathrm{m}$ ) by possible self-absorption problems. We note that the reduction or not (by a factor of two) the flux of the [CII] $158 \mu \mathrm{m}$ line to account for the background contribution (see Sect. 4), does not significantly alter the data points in Fig. 6. These circumstances make the plot in Fig. 6 more reliable than that in Fig. 5. In Fig. 6 all the HAEBE showing molecular emission are depicted, although three of them (R CrA, IRAS 12496 and $\mathrm{LkH} \alpha$ 234) were discussed in Paper II. The newly considered objects (TY CrA, BD $+40^{\circ} 4124$ and MWC 361) trace densities of the order of $10^{6} \mathrm{~cm}^{-3}$ or less and $G_{\mathrm{o}}$ between $10^{3}$ and $10^{4}$, confirming the association between molecular emission and high density condensations. Support to this finding comes from independent observations: according to $\mathrm{CO}$ data in the $\mathrm{CrA}$ region, the column density maximum is close to the position of TY CrA where $n>10^{4} \mathrm{~cm}^{-3}$ (Harju et al. 1993); clumpy 


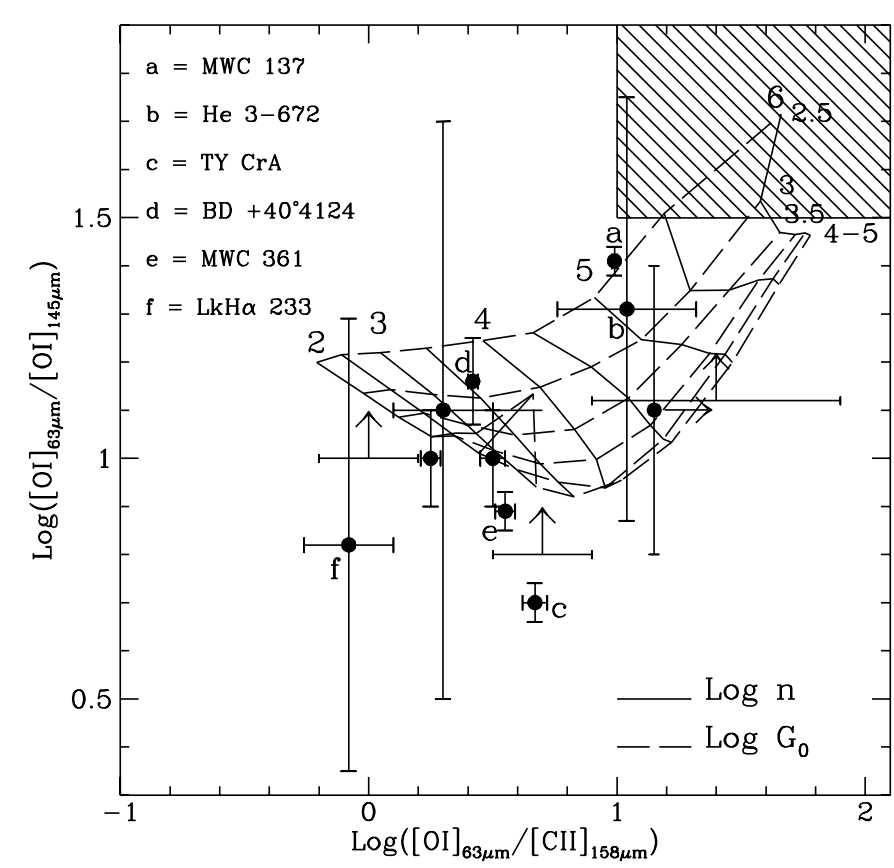

Fig. 5. Observed line ratios superimposed to PDR models by Kaufman et al. (1999). The hatched area identifies the locus pertaining to the J-shock model predictions.

photodissociation regions (PDRs) with high density filaments $\left(n \sim 10^{5}-10^{6} \mathrm{~cm}^{-3}\right)$ are expected to be immersed in a more diffuse medium around MWC 361 (Fuente et al. 2000); a clumpy PDR (with $n \sim 10^{6} \mathrm{~cm}^{-3}$ ) is proposed by van den Ancker et al. (2000) as the unique alternative to account for their observations of the $\mathrm{BD}+40^{\circ} 4124$ region. If molecular emission originates close to density peaks it could be associated, rather than to the HAEBE itself, to a nearby and more embedded companion of the kind that has sometimes been discovered in HAEBE neighbourhoods (e.g. Font et al. 2001).

Apart from photodissociation, a deeper discussion on the remaining alternatives is done in Paper II. Here we remember that molecular emission can also occur in $C$ - or $J$-shocks. However, $J$-shocks at low values of the pre-shock density $\left(n_{0} \simeq\right.$ $10^{4} \mathrm{~cm}^{-3}$ ) predict $L_{[\mathrm{OI}]} / L_{\mathrm{CO}} \gg 1$, while we have for TY CrA, $\mathrm{BD}+40^{\circ} 4124$ and MWC 361 quite low values for the $L_{[\mathrm{OI}]} / L_{\mathrm{CO}}$ ratio $(0.7,0.2,0.3$, respectively). According to both $C$-shocks and $J$-shocks at high values of the pre-shock density $\left(n_{0} \simeq\right.$ $10^{6} \mathrm{~cm}^{-3}$ ), water should be the main coolant with a predicted cooling ratio $L_{\mathrm{H}_{2} \mathrm{O}} / L_{\mathrm{CO}} \sim 10$, clearly in contrast with the absence of water emission in all the 26 HAEBE. In conclusion, the combined diagnostic provided by fine structure and molecular line emission tends to favour the photodissociation mechanism possibly operating in a clumpy medium.

\subsection{Ionised lines}

Fine structure lines from different ions are detected, among the objects presented here, only toward $\mathrm{BD}+40^{\circ} 4124$ in form of [OIII] 52 and $88 \mu \mathrm{m}$ and [NII] $122 \mu \mathrm{m}$ transitions. This circumstance is not unexpected since this source belongs to the only $\mathrm{OB}$ association among our sample. Previously, van den Ancker et al. (2000), by using the intensity ratio of the [OIII] lines, found they are formed in a HII region with an electron density $n_{\mathrm{e}}=0.6-2.2 \times 10^{2} \mathrm{~cm}^{-3}$. Their paper provides a deep study of the $\mathrm{BD}+40^{\circ} 4124$ group, also including the nearby objects and the relative SWS observations. Here we have also detected the [NII] $122 \mu \mathrm{m}$ line which is a typical tracer of low ionisation and low density material. From the ratio $[\mathrm{CII}] 158 \mu \mathrm{m} /[\mathrm{NII}] 122 \mu \mathrm{m} \simeq 16$, we derive, by using the Petuchowsky \& Bennet model (1993), a lower value of $n_{\mathrm{e}}=25 \mathrm{~cm}^{-3}$, which indicates the presence of an electron density gradient moving away from the central object.

\subsection{About the ratio [OI]63 $\mu \mathrm{m} /[\mathrm{OI}] 145 \mu \mathrm{m}$}

Considering all the 26 HAEBE we note that, when the $145 \mu \mathrm{m}$ line is detected, the $[\mathrm{OI}] 63 \mu \mathrm{m} /[\mathrm{OI}] 145 \mu \mathrm{m}$ line ratios range between 2 and 10 with few exceptions at larger values (see Paper I and Fig. 5). Conversely, the models either simply based on density and temperature conditions (e.g. Watson 1984) or oriented to specific excitation mechanisms (PDR, $J$-, $C$-shocks), all predict larger values of this ratio typically in the range 20-100 or more; in this framework, the observed values represent systematically quite marginal cases. The unconsistency between model predictions and observed [OI] line ratios is emphasized by independent evidences coming from all the authors who have studied star forming regions with ISO-LWS data (e.g. Fuente et al. 2000; Liseau et al. 1999; Saraceno et al. 1998). The only exception is represented by the class of the HerbigHaro objects, whose observed ratios are between 15 and 25 (e.g. Molinari et al. 2001; Nisini et al. 1996). In Paper I we have explained the low ratios as due to the presence of foreground cold OI responsible for an absorption at $63 \mu \mathrm{m}$ and thus producing a net decrease of the $63 \mu \mathrm{m}$ flux emitted by the source. But $A_{V} \geq 5$ mag is required to justify such an absorption, therefore this explanation cannot be extended to the objects presented in this paper because those having 63/145 ratio less than 10 have all $A_{V}<2.5 \mathrm{mag}$. A possible interpretation for the recurrent low values could be related to the optical depth of both lines. Values between 2 and 10 can be obtained in a gas with a temperature between 100 and $500 \mathrm{~K}$, if both lines are optically thick (see e.g. Fig. 2 of Tielens \& Hollenbach 1985). By assuming an oxygen abundance $X(\mathrm{O}) / X(\mathrm{H})=3.2 \times 10^{-4}$ (Meyer et al. $1998)$ with all the oxygen in the OI form and a dispersion velocity of $2 \mathrm{~km} \mathrm{~s}^{-1}$, we obtain that the required column densities to make the $\tau_{63 \mu \mathrm{m}}, \tau_{145 \mu \mathrm{m}} \geq 1$ are $3.3 \times 10^{22}$ and $2.2 \times 10^{22} \mathrm{~cm}^{-2}$ at 100 and $500 \mathrm{~K}$, respectively. These values correspond to $A_{V}=17$ and $11 \mathrm{mag}$, by adopting the total-to-selective extinction ratio $R=3$. Since $R=5$ seems more appropriate for grains around HAEBE (Pezzuto et al. 1997), the $A_{\mathrm{V}}$ values move in the range $27-18 \mathrm{mag}$. These conditions (temperatures of about 100-500 K and high optical depths) are typical of compact structures such as disks or clumps around the central object (Burton et al. 1990). A rough estimate of the size $l$ of the emitting region, can be derived from the ratio between the column and the volume density $(l \simeq N / n \mathrm{~cm})$, if homogeneity and spherical symmetry are assumed as for the circumstellar environment. By adopting the clump densities derived from Fig. 6 


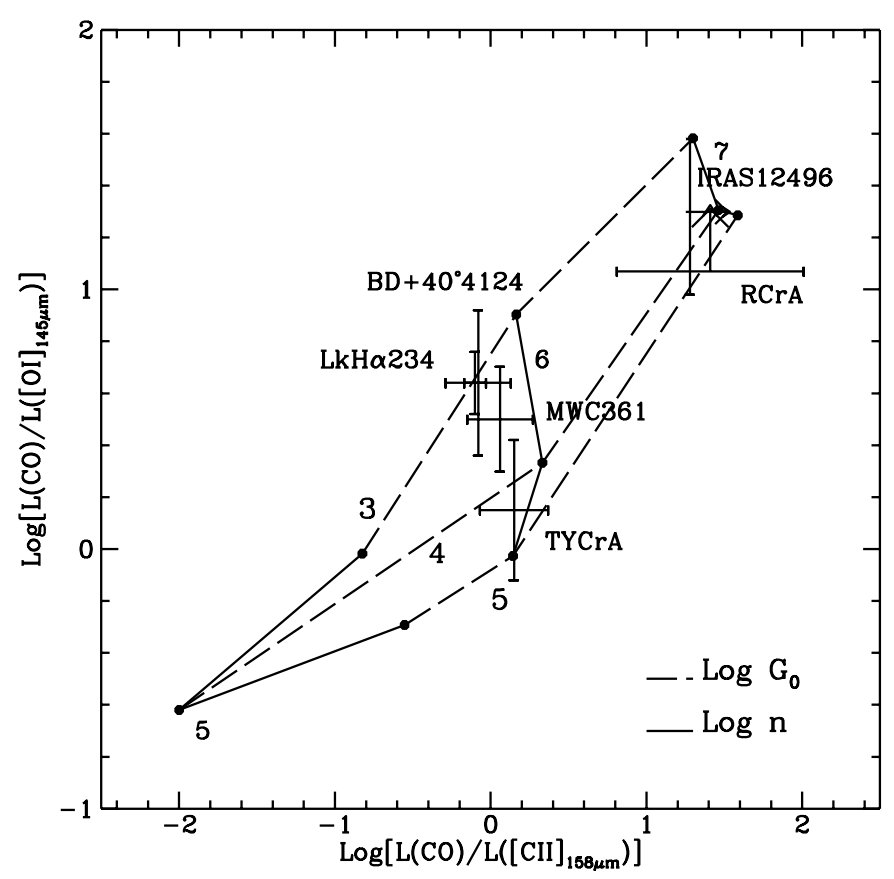

Fig. 6. Observed ratios between molecular CO lines and fine structure [OI] $145 \mu \mathrm{m}$ and [CII] $158 \mu \mathrm{m}$ superimposed on clumpy PDR models.

and the column density required to have $\tau_{[\mathrm{OI}] \text { lines }} \geq 1$, we obtain $l \simeq 2000 \mathrm{AU}$, which seems an upper limit given the typical size of the clumps. Smaller values of $l$ can be derived by assuming that only the $63 \mu \mathrm{m}$ line is optically thick, while the $145 \mu \mathrm{m}$ one is still optically thin. This situation obviously implies $A_{V}$ values smaller than 27-18 mag. Therefore the low observed values of the 63/145 line ratio seem to be consistent with the presence of high column densities (roughly between 10 and $20 A_{V}$ ) of a gas at $100-500 \mathrm{~K}$, conditions which are not explored by the current models of PDR illuminated by interstellar radiation. In these models the observer is expected to see the illuminated emitting zone where temperatures between 500 and $100 \mathrm{~K}$ pertain to a gas with low optical depth ( $A_{V} \approx 2 \mathrm{mag}$ ). On the contrary, when the illuminating source (with an intensity likely stronger than that of the interstellar field) lies in the opposite side, the observer could see the emitting gas (at 100-500 K) at substantially higher column densities.

Finally we note that in the considered case (i.e. $63 \mu \mathrm{m}$ line optically thick) the $63 / 145$ ratio should not represent a suitable diagnostic tool to trace the density, but other line ratios, which include only the $145 \mu \mathrm{m}$ transition, seem more appropriate to that scope. This is well demonstrated by the plot in Fig. 6, while we fail to obtain a meaningful matching between observations and models by using the $63 \mu \mathrm{m}$ line in the same plot.

\subsection{An evolutionary trend}

Since our complete ISO-LWS sample (26 HAEBE out of 108) can be considered representative enough of the entire class, we have tried to derive some general trend to be compared with similar behaviours from classes of younger objects. We have computed the far IR luminosity $\left(L_{\mathrm{FIR}}\right)$ as due to all line

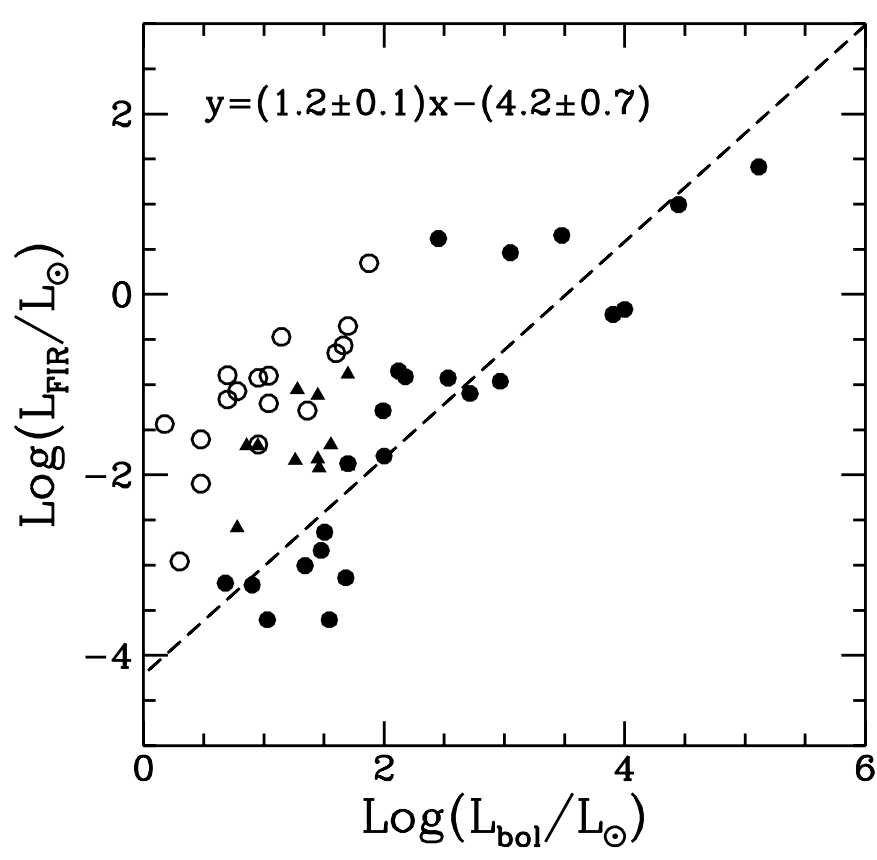

Fig. 7. Far IR lines luminosity $v s$. bolometric luminosity of the central star. The equation representing the best linear fit to the points (dashed line) is given in the upper part. HAEBE are represented as filled circles, while younger Class 0 and Class I objects (see text) are indicated with triangles and open circles, respectively.

emission contributions (fine structure line emission and total molecular cooling). In Fig. 7 these values are plotted as a function of the bolometric luminosity of the central object; the straight line, whose equation is given in the upper part of the Figure, represents the best linear fit to the points. We are aware of the distance bias built in the lum-lum plots which present (in the Log form) a linear correlation with unitary angular coefficient, thus we intend just to point out that the intercept on the $y$-axis is located at a $L_{\mathrm{FIR}}$ value of about $10^{-4}$. A similar plot for the younger Class 0 objects (Nisini et al. 2002 , here depicted as triangles) indicates $L_{\mathrm{FIR}} \simeq 10^{-2}$ as the intercept value. An intermediate value of $10^{-3}$ is attributable to the Class I objects (open circles), which are expected to be somehow younger than HAEBE and approaching to their evolutionary stage. The mechanisms invoked to account for the behaviour of the HAEBE stars and of the other objects are completely different: photodissociation due to FUV stellar photons for the former stars and shock excitation for the latter sources, where the $L_{\mathrm{FIR}}$ dependence on $L_{\mathrm{bol}}$ is related to the relationship between accretion rate $\left(\dot{M}_{\text {acc }}\right)$ and the mass loss rate $\left(\dot{M}_{\text {out }}\right)$. However it is worthwhile noting how $L_{\mathrm{FIR}}$ is a decreasing fraction of $L_{\mathrm{bol}}$ while the evolution goes on, although the different phases obey to different physical mechanisms. The conversion of the bolometric luminosity of the central object into far IR line cooling in the circumstellar envelope occurs at progressively lesser efficiency while time elapses. This fact extends to the far IR gas cooling the well consolidated statement according to which the relevance of the circumstellar vs. stellar properties diminishes during the pre-stellar evolution. 


\section{Conclusions}

The main points of this work can be summarized as follows:

- The ISO-LWS archive has been systematically searched to obtain a complete far IR spectrophotometric survey of HAEBE stars: 15 new objects complement our original list of 11, leading to a final catalog of 26 objects, which represent about $25 \%$ of all the known HAEBE. Far IR spectra (from 45 to $200 \mu \mathrm{m}$ at low resolution $\mathcal{R} \simeq 200$ ) of the new HAEBE stars have been analysed following the same approach as in our prior papers.

- [OI] $63 \mu \mathrm{m}$ and [CII] $158 \mu \mathrm{m}$ lines are observed in many of the investigated objects (with a detection rate of $73 \%$ and $85 \%$, respectively), while the [OI] $145 \mu \mathrm{m}$ is more difficultly detected (42\%). Molecular lines in form of $\mathrm{CO}$ high- $J$ rotational transitions are detected toward three sources and are associated to local density peaks (23\%).

- A new class of ISO-LWS spectra of HAEBE has been put into evidence: that constitued by low luminosity objects $\left(L_{\text {bol }} \lesssim 30 L_{\odot}\right)$ in whose spectra we do not detect any gas feature. In such cases, the weak [CII] $158 \mu \mathrm{m}$ emission is likely a contribution due to a diffuse component not related with the star itself.

- Line intensities and intensity ratios have been compared with model predictions and we find that photodissociation by stellar photons, active in a clumpy medium, is the prevalent excitation mechanism.

- In a large number of cases the ratio [OI]63 $\mu \mathrm{m} /[\mathrm{OI}] 145 \mu \mathrm{m}$ is anomalously less than expected. Optical depth effects are discussed as the most likely reason of these values: maybe some reconsideration of the PDR models toward more realistic morphologies of the emitting region could reconcile the observed discrepancy.

- An evolutionary trend is clearly recognizable in the ratio $L_{\mathrm{FIR}} / L_{\mathrm{bol}}$ between the luminosity due to all the line emission contributions and the bolometric luminosity of the central object. This ratio, which is about $10^{-4}$ for HAEBE, is smaller than that of younger objects, thus it decreases while the evolution goes on.

- This catalog serves as spectroscopic database for future space missions, when it will be possible to increase spatial resolution and sensitivity. The corresponding increase in the detection rate of both the [OI] $145 \mu \mathrm{m}$ and molecular emission can be used for confirming the view presented here.

\section{References}

Benedettini, M., Nisini, B., Giannini, T., et al. 1998, A\&A, 339, 159 Benedettini, M., Pezzuto, S., Giannini, T., et al. 2001, A\&A, 379, 557 Bouwman, J., de Koter, A., van den Ancker, M. E., \& Waters, L. B. F. M. 2000, A\&A, 360, 213

Burton, M. G., Hollenbach, D. J., \& Tielens, A. G. G. M. 1990, ApJ, 365,620
Clegg, P. E., Ade, P. A. R., \& Armand, C. 1996, A\&A, 315, L38

Corporon, P., Ceccarelli, C., \& Lagrange, A.-M. 1999, in The Universe as seen by ISO - ESA-SP 427, ed. P. Cox, \& M. Kessler, 297

Creech-Eakman, M. J., Chiang, E. I., Joung, R. M. K., Blake, G. A., \& van Dishoeck, E. F. 2002, A\&A, 385, 546

Draine, B. T., Roberge, W. G., \& Dalgarno, A. 1983, ApJ, 264, 485

Elia, D., Campeggio, L., Strafella, F., et al. 2002, A\&A, submitted

Font, A. S., Mitchell, G. F., \& Sandell, G. 2001, ApJ, 555, 950

Fuente, A., Martin-Pintado, J., Rodriguez-Fernández, N. J., Cernicharo, J., \& Gerin, M. 2000, A\&A, 354, 1053

Giannini, T., Lorenzetti, D., Tommasi, E., et al. 1999, A\&A, 346, 617 (Paper II)

Harju, J., Haikala, L. K., Mattila, K., et al. 1993, A\&A, 278, 569

Hollenbach, D., \& McKee, C. F. 1989, ApJ, 342, 306

Hollenbach, D., Takahashi, T., \& Tielens, A. G. G. M. 1991, ApJ, 377 , 192

Kaufman, M. J., Wolfire, M. G., Hollenbach, D. J., \& Luhman, M. L. 1999, ApJ, 527, 795

Kessler, M., Steinz, J. A., \& Anderegg, M. E. 1996, A\&A, 315, L27

Liseau, R., White, G., Larsson, B., et al. 1999, A\&A, 344, 342

Lorenzetti, D., Tommasi, E., Giannini, T., et al. 1999, A\&A, 346, 604 (Paper I)

Malfait, K., Waelkens, C., Waters, L. B. F. M., et al. 1998, A\&A, 332, L25

Meeus, G., Waters, L. B. F. M., Bouwman, J., et al. 2001, A\&A, 365, 476

Meyer, D. M., Jura, M., \& Cardelli, J. A. 1998, ApJ, 493, 222

Molinari, S., Noriega-Crespo, A., \& Spinoglio, L. 2001, ApJ, 547, 292

Nisini, B., Giannini, T., \& Lorenzetti, D. 2002, ApJ, in press

Nisini, B., Lorenzetti, D., Cohen, M., et al. 1996, A\&A, 315, L321

Palla, F., Testi, L., Hunter, T. R., et al. 1995, A\&A, 293, 521

Petuchowsky, S. J., \& Bennett, C. L. 1993, ApJ, 405, 591

Pezzuto, S., Strafella, F., \& Lorenzetti, D. 1997, ApJ, 485, 290

Pilbratt, G. L. 2001, in The promise of the Herschel Space Observatory - ESA-SP 460, ed. G. L. Pilbratt, J. Cernicharo, A. M. Heras, T. Prusti, \& R. Harris, 13

Saraceno, P., Nisini, B., Benedettini, M., et al. 1998, Proc. of Star Formation with the Infrared Space Observatory, ed. J. L. Yun, \& R. Liseau, ASP Conf. Ser. 132, 233

Swinyard, B. M., Clegg, P. E., Ade, P. A. R., et al. 1996, A\&A, 315, L43

Thé, P. S., de Winter, D., \& Pérez, M. R. 1994, A\&AS, 104, 315

Thi, W. F., van Dishoeck, E. F., Blake, G. A., et al. 2001, ApJ, 561, 1074

Tielens, A. G. G. M., \& Hollenbach, D. 1985, ApJ, 291, 722

van den Ancker, M. E., Wesselius, P. R., \& Tielens, A. G. G. M. 2000, A\&A, 355, 194

van den Ancker, M. E., de Winter, D., \& Tjin A Djie, H. R. E. 1998, A\&A, 300, 145

Waelkens, C., Waters, L. B. F. M., de Graauw, M. S., et al. 1996, A\&A, 315, L245

Watson, W. D. 1984, in Galactic and Extragalactic Infrared Spectroscopy, ed. M. F. Kessler, \& J. P. Phillips (Dordrecht: Reidel), 195

Wesselius, P. R., van den Ancker, M. E., Young, E. T, et al. 1996, A\&A, 315, L197 